\title{
Functional electrical stimulation reduces pain and shoulder subluxation in chronic post-stroke patients?
}

\author{
Willian Vasconcellos da Silva', Gabriele Natane de Medeiros Cirne' Edson Meneses da Silva Filho', \\ Enio Walker Azevedo Cacho', Johnnatas Mikael Lopes², Roberta de Oliveira Cacho', Marina Pegoraro Baroni .
}

\begin{abstract}
Background: Shoulder subluxation is a common complication of cerebral vascular accident (stroke) and the use of Functional Electrical Stimulation (FES) within the rehabilitation process is extremely important. Objective: To analyze the therapeutic effects of FES in the treatment of chronic shoulder subluxation in post-stroke patients. Method: This is a case study of patients with radiologically subluxation confirmed, who were randomly divided into two groups: Control Group (CG) and Treatment Group (TG). Patients were assessed before and after treatment and at the 2-month follow-up. The assessment consisted of the modified Ashworth scale; passive goniometry; Fugl-Meyer scale; McGill pain questionnaire and evaluation of shoulder subluxation by radiography. The CG did not receive physiotherapeutic intervention; and TG underwent 20 sessions of motor kinesiotherapy and FES associated with functional exercises with a total duration of 1 hour, three times a week, for 7 weeks. The data were analyzed descriptively. Results: The mean age of CG participants was $82.5 \pm 1.5$ years and of the TG was $70.5 \pm 13.5$ years. All of them were retired, sedentary, non-smokers/alcoholics and had hemiparesis on the left side. There was an approximate increase of $10^{\circ}$ for most joint movements of the shoulder, improvement in McGill scale scores and reduction of shoulder subluxation in TG patients. Conclusion: FES associated with functional movements was effective in reducing the degree of subluxation of the shoulder joint and decreased pain in subjectsin the chronic phase of the post-stroke.

Keywords: Stroke, Shoulder Dislocation, Electric Stimulation, Pain.
\end{abstract}

\section{INTRODUCTION}

The adult brain needs a supply of 150 grams of glucose and 72 liters of oxygen every hour, though it does not store these substances. In this way, any dysfunction in the cerebral vascularization can cause cerebral damages ${ }^{(1)}$. One of the most common examples of cerebral vascular damage is stroke resulting from the sudden loss of neurological functions caused by an interruption of blood flow (ischemic) or by an extravasation of blood in the intracerebral space (hemorrhagic) ${ }^{(1-3)}$. The most common primary dysfunctions are linked to paresis or folding of the hemi body contralateral to the central lesion, and include muscle strength deficits, changes in muscle tone (hypertonia), loss of coordination and balance, abnormal synergistic patterns, and functional disability. Among the complications secondary to stroke, the following can be highlighted deep vein thrombosis, shoulder pain and subluxation ${ }^{(1-6)}$. Shoulder or glenohumeral subluxation is a complication of stroke with prevalence in up to $81 \%$ of cases $^{(7)}$. The articulation of the shoulder presents in its anatomical structure the humeral head larger than the glenoid fossa, thus making it the most mobile and least stable of all joints in the human body ${ }^{(7-8)}$. To aid in this size difference and increase its coaptation, the glenoid lip, which is a fibrous ring formed by fibrocartilage, surrounds the glenoid cavity with the function of giving greater stabilization ${ }^{(7)}$. In addition, the shoulder joint relies on the dynamic stability provided by the rotator cuff and deltoid muscles ${ }^{(7-9)}$.

After the stroke, there may be a compromise in the cortical-spinal pathway of the subjects, which is responsible for the voluntary movement of the body, causing loss of voluntary movements on the contralateral side of the injury, reducing the dynamic stabilizers acting, causing joint instability and favoring a possible subluxation of the glenohumeral joint ${ }^{(1-3)}$. This condition leads to the appearance of a protrusion between the acromion and the humeral head, which can be identified by palpation or radiographic examination ${ }^{(10-13)}$. Shoulder subluxation may lead to situations of movement limitations, adhesive capsulitis, brachial plexus lesions, rotator cuff lesions and glenohumeral joint pain ${ }^{(4)}$. These clinical conditions may impair the functional recovery of post-stroke patients and constitute a major challenge to the rehabilitation program 
for these persons ${ }^{(11-15)}$. Within the rehabilitation process, the use of Functional Electrical Stimulation (FES) stands out. Several studies have shown that FES therapy is effective for the activation of the shoulder stabilizing muscles, improving glenohumeral joint congruence, functional improvement and reduction of pain in patients with post-stroke glenohumeral subluxation $^{(8-13)}$. However, the studies are inconclusive and did not analyze the effects of FES on spasticity, range of motion (ROM) and motor functional assessment. Considering the assumptions presented, this study aimed to analyze the therapeutic effects of FES in the treatment of chronic shoulder subluxation in post-stroke patients.

\section{METHOD}

This is a case study with blind evaluation conducted at the Faculty of Health Sciences of the Trairi - FACISA, Santa Cruz Rio Grande do Norte, Brazil. It was approved by the Research Ethics Committee of the Federal University of the Rio Grande do Norte (CEP/UFRN), with protocol number 166/11, CAAE no 0329.0.051.000-11. The inclusion criteria were clinical diagnosis of ischemic or hemorrhagic stroke for more than one year, shoulder subluxation confirmed by radiography, not using analgesics and presenting disproportionate hemiparesis with brachial predominance. Patients with a pacemaker, orthopedic and/or rheumatologic disease in the shoulder, comprehension aphasia, lack of sensitivity in the shoulder region, clinical instability (uncontrolled hypertension and diabetes mellitus), and cognitive deficit measured by Mini-Mental State Examination (MMSE) were excluded ${ }^{(16)}$. After signing the Informed Consent Term (ICF), the participants were randomly assigned to 2 groups: treatment group (TG) and control group (CG). Each group consisted of two patients who underwent blind evaluations five days before the intervention, at the end of the treatment protocol and after 2 months of the end. The evaluation consisted of anamnesis, assessment of shoulder subluxation, assessment of spasticity, pain assessment, ROM assessment, motor evaluation and cognitive evaluation. The anamnesis contained identification data (name, address, gender), history of the current disease, history of previous diseases, use of medications, physical examination, among others.

To evaluate the subluxation of the shoulder, a radiographic examination was performed on the anterior-posterior incidence of the subluxated glenohumeral joint and the joint not affected for comparison and diagnosis, with the patient in the orthostatic position. To identify the degree of subluxation, a millimeter ruler was used to determine the distance (d) from the inferior border of the glenoid fossa to the inferior line between the anatomic neck of the humeral head, being considered subluxation of the shoulder a distance greater than seven millimeters ${ }^{10}$, as shown in figure 1 . Spasticity was verified using the Modified Ashworth Scale (MAS) in the external rotator muscle groups and vertical abductors of

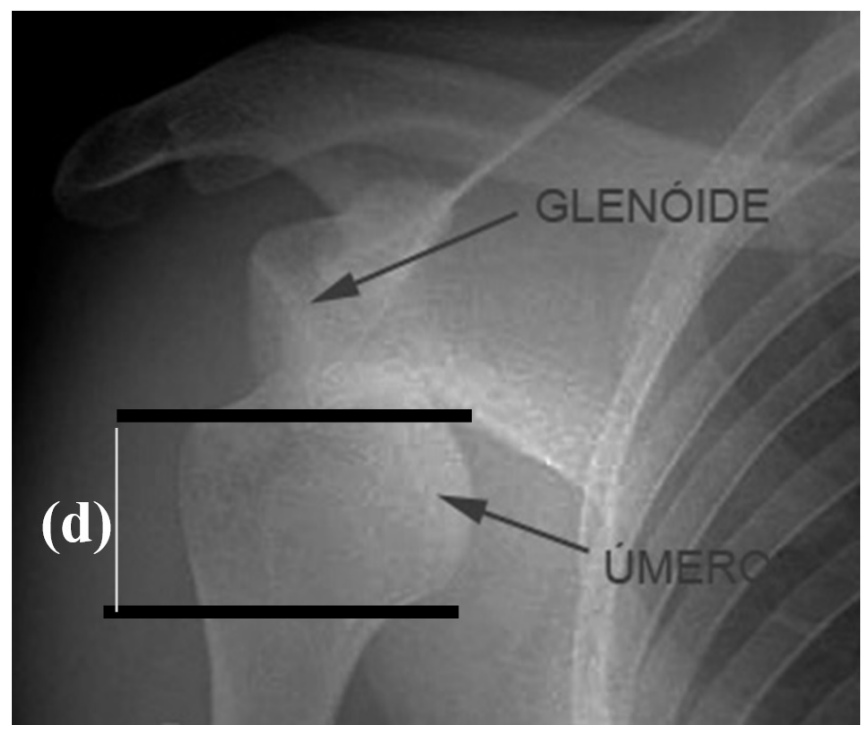

Figure 1: Measurement of shoulder subluxation. Note: (d): distance; Glenóide: Glenoid; Úmero: Humerus.

the shoulder(2). Pain intensity was assessed using the McGill Pain Questionnaire ${ }^{(17)}$. The passive ROM of the shoulder was measured with a manual goniometer in the movements of flexion, extension, abduction, aduction and rotation of the shoulder, according with the protocol proposed previously ${ }^{(18)}$. The motor evaluation of the upper limb was performed using the protocol of physical performance of Fugl-Meyer ${ }^{(19-20)}$.

The therapeutic intervention consisted of FES associated with kinesiotherapy. Twenty sessions were performed, with a total duration of 1 hour, three times a week, for 7 weeks. Kinesiotherapy consisted of techniques of upper limb mobilization, weight discharging and passive stretching in the rhomboid, large dorsal, pectoralis major, trapezius and biceps brachial muscles until a modulation of the patient's spastic tone was obtained, when necessary. The FES equipment used was the FES Vif 995 Four of the Quark ${ }^{\circledR}$ brand (Quark Medical, Piracicaba, SP, Brazil). After cleaning the region with $70 \%$ alcohol, the electrodes were positioned at the motor points of the supraspinal and posterior deltoid muscles of the subluxated shoulder, and fixed with tape. The parameters were: frequency of $30 \mathrm{~Hz}$; pulse duration time of $300 \mu \mathrm{s}$, T-on of 15 seconds, T-off of 30 seconds, treatment time of 45 minutes and amplitude according to motor threshold. Simultaneously with the passage of the current on the T-on, the patient was asked to perform active functional abduction and flexion movements of the shoulder, such as simulating combing / washing hair and reaching / carrying objects above the head. When the active realization of the functional movements was not possible, the movements were performed passively or actively-assisted by the therapist. The presentation of the data was done in a descriptive way using Excel ${ }^{\circledR}$ software. 


\section{RESULTS}

The sample consisted of 4 participants with mean age of $82.5 \pm 1.5$ years for $C G$ and $70.5 \pm 13.5$ years for TG. Both CG and TG patients were retired, had left-sided hemiparesis, sedentary, non-smokers and non-alcoholics. The CG and TG patient profiles are described in Table 1. The clinical evolution of the pain, subluxation and motor impairment parameters are shown in Table 2 . It can be observed that there is a

Table 1. Profile of the participants.

\begin{tabular}{|c|c|c|c|c|}
\hline \multirow{2}{*}{ Variables } & \multicolumn{2}{|c|}{ Control Group } & \multicolumn{2}{|c|}{ Treatment Group } \\
\hline & Patient 1 & Patient 2 & Patient 3 & Patient 4 \\
\hline Age (years) & 81 & 84 & 57 & 84 \\
\hline Scholarity & Illiterate & $\begin{array}{l}\text { Incomplete } \\
\text { primary }\end{array}$ & $\begin{array}{l}\text { Complete } \\
\text { High School }\end{array}$ & $\begin{array}{c}\text { Incomplete } \\
\text { primary }\end{array}$ \\
\hline Stroke Type & Hemorrhagic & Ischemic & Both & Ischemic \\
\hline $\begin{array}{l}\text { Hemisphere } \\
\text { affected by } \\
\text { stroke }\end{array}$ & Left & Left & Left & Left \\
\hline $\begin{array}{l}\text { Subluxed } \\
\text { Shoulder }\end{array}$ & Left & Left & Left & Left \\
\hline MMSE Score & 28 & 28 & 26 & 28 \\
\hline
\end{tabular}

Note: MMSE: Mini-Mental State Examination.

Table 2. Quantitative analysis of the evolution of the clinical condition regarding pain, degree of subluxation and motor impairment.

\begin{tabular}{|c|c|c|c|c|c|c|c|c|c|}
\hline \multirow{2}{*}{ Patients } & \multicolumn{3}{|c|}{ McGill } & \multicolumn{3}{|c|}{$\begin{array}{l}\text { Subluxation degree } \\
(\mathrm{mm})\end{array}$} & \multicolumn{3}{|c|}{ Fugl-Meyer Scale } \\
\hline & Pre & Post & Ret & Pre & Post & Ret & Pre & Post & Ret \\
\hline CG - 1 & 40 & 41 & 40 & 11 & 11 & 11 & 0 & 0 & 0 \\
\hline CG - 2 & 45 & 42 & 48 & 10 & 10 & 10 & 0 & 0 & 0 \\
\hline TG -3 & 30 & 0 & 0 & 11 & 7 & 7 & 28 & 28 & 30 \\
\hline TG -4 & 48 & 0 & 0 & 10 & 5 & 5 & 0 & 0 & 0 \\
\hline
\end{tabular}

Note: Pre: pre-treatment; Post: post-treatment; Ret: Retention test; CG: control group; TG: treatment group; mm: millimeters. reduction from pre- to post-treatment and retention test in the pain and subluxation variables only in TG patients. Table 3 shows the absolute values for measures of joint spasticity and ROM. There are considerable improvements in the degree of shoulder joint amplitude only for TG patients.

\section{DISCUSSION}

In the present study were evaluated the pain, subluxation, ROM, spasticity and motor function of the subluxated shoulder of four women after chronic stroke. After twenty sessions of physiotherapeutic care, a significant decrease of pain was observed, with the two patients of the TG obtaining a null score in the McGill questionnaire and a decrease in subluxation, between 4 and $5 \mathrm{~mm}$. This reduction in shoulder subluxation was sufficient for patients to reach the normal range for the distance from the inferior border of the glenoid fossa to the inferior line of the anatomical neck of the humeral head ${ }^{(10)}$. These findings corroborate with other studies ${ }^{(12,21)}$. In a study 50 patients with shoulder subluxation were divided in control group (conventional kinesiotherapy) and treatment group (kinesiotherapy and FES), with parameters of the FES with frequency of $36 \mathrm{~Hz}$; pulse duration of $250 \mu \mathrm{s}$; T-on of 30 seconds; T-off of 15 seconds, 1 hour/day, in a total of 20 sessions. The authors observed a significant reduction of pain and degree of subluxation $(p<0.05)$ in the treatment group compared to the control group ${ }^{(12)}$. In another study the FES was used in the supraspinal muscle and posterior deltoid fibers in eight patients, with FES parameters of a frequency of $12 \mathrm{~Hz}$; pulse duration of $200 \mu \mathrm{s}$; T-on of 10 seconds; T-off of 10 seconds, every day of the week for 6 hours/day over six weeks. At the end of treatment there was a decrease in pain and a decrease in shoulder subluxation ${ }^{(21)}$. The improvement of the shoulder subluxation and pain can be justified by the activation of the dynamic stabilizing muscles of the shoulder through FES. The activation of this musculature causes a greater coaptation of the humerus in the glenoid fossa, thus

Table 3. Quantitative analysis of the evolution of the clinical condition regarding spasticity and range of joint motion.

\begin{tabular}{|c|c|c|c|c|}
\hline \multirow{3}{*}{ Variables* } & \multicolumn{2}{|c|}{ Control Group (CG) } & \multicolumn{2}{|c|}{ Treatment Group (TG) } \\
\hline & Patient 1 & Patient 2 & Patient 3 & Patient 4 \\
\hline & Pre/Post/Ret & Pre/Post/Ret & Pre/Post/Ret & Pre/Post/Ret \\
\hline MAS - rot ext & $3 / 3 / 3$ & $1 / 1 / 1$ & $1 / 1 / 1$ & $1 / 1 / 1$ \\
\hline MAS - abducers & $1 / 1 / 1$ & $1 / 1 / 1$ & $1 / 1 / 1$ & $1 / 1 / 1$ \\
\hline ROM - flexors & 700 / 70ㅇ / 70은 & $56 ㅇ$ / 52 / 500 & 112ㅇ / 108ㅇ / 122응 & 500 / 64ㅇ / 64은 \\
\hline ROM - extenders & 240 / 25이 / 24음 & $38^{\circ}$ / 36ㅇ / 38은 & 40 / 50 / 50ㅇ & $38 ㅇ / 50 ㅇ / 50$ \\
\hline ROM - adductors & 18ㅇ / 18ㅇ / 18우 & 240 / 24ㅇ / 240 & $180 / 28 \circ / 38$ & $24 \circ / 30 \circ / 30 \circ$ \\
\hline ROM - abducers & 840 / $83^{\circ}$ / 84 & $58^{\circ}$ / $58^{\circ}$ / $58^{\circ}$ & 94 / 120ㅇ / 128음 & $58 ㅇ / 80 ㅇ / 80 ㅇ$ \\
\hline ROM - rot int & $72 \circ / 70 \div / 72 \circ$ & 90 / 90ㅇ / 90음 & $80 ㅇ$ / 88ㅇ / 90은 & 90 / 90ㅇ / 90 \\
\hline ROM - rot ext & 8ㅇ / 8ㅇ / 8ㅇ & 10ㅇ / 8ㅇ / 10은 & $44^{\circ}$ / 58ㅇ / 58은 & 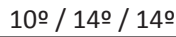 \\
\hline
\end{tabular}

Note: Pre: pre treatment; Post: Post Treatment; Ret: Retention Test; CG: Control Group; TG: Treatment Group; Rot: rotators; Int: internal; Ext: external; MAS: Modified Ashworth Scale; ROM: Range Of Motion. *all variables refer to the subluxated shoulder joint. 
reducing the subluxation of the shoulder and consequently generating lower joint stress and decrease of the pain ${ }^{(10)}$. There was no improvement in motor impairment assessed by the Fugl-Meyer Scale. This can be explained by the previous severe impairment of the patients (score of zero on the Fugl-Meyer Scale in three of the four patients) and the chronicity of the stroke, which are associated with a worse recovery prognosis. Similar to the present study, the result of another that observed a worse proportion of improvement in that patient with higher motor impairment of the upper limb evaluated by Fugl-Meyer scale ${ }^{(11)}$. There is evidence that therapeutic exercises influence the spontaneous recovery and adaptability of brain injury ${ }^{(22-23)}$. However, it is seen that the best result in neuro-rehabilitation occurs when the intervention is precocious, of high intensity, with execution of tasks with specific objectives and carried out in an active way, coordinated by a multiprofessional team ${ }^{(24)}$. Another factor that contributes to the functional deficit of the post-stroke patient is spasticity, which alters motor control and may impair functional performance. This is evidenced in this study by the maintenance of the spasticity evaluation result during the three evaluations. Two factors are believed to explain this result. The first is that the parameters used by the FES were established in order to avoid muscle fatigue, and one of the techniques of FES use to reduce spasticity is precisely to cause muscle fatigue with high frequencies, and time-off shorter than the time-on ${ }^{(25-26)}$. In addition, MAS evaluates tonus by muscle groups, however in this study FES was applied to only one of the muscles of the shoulder joint. Therefore, there may have been some reduction of muscle tone, but not enough to be detected in the manual examination.

There was an improvement in the ROM of the shoulder in the TG in the joint movements that the flexors and extensors of the shoulder acted (around $10^{\circ}$ of the pretreatment for the retention test) and shoulder abductors (34으 for patient 3 and $22^{\circ}$ for the patient 4 , from the pretreatment to the retention test), which corroborates with another study ${ }^{(21)}$. Apparently, assisted active and active movement exerted by the FES and functional exercises promoted the production of synovial fluid in the joints and improves the capsular, ligament and muscle stretching, allowing gains in the dynamic movement ${ }^{(11)}$. In another research, the FES was used in patients with shoulder subluxation who had acute and chronic stroke for six weeks and there was improvement in shoulder subluxation only in patients who had acute stroke ${ }^{(27)}$. However, other studies show that, even in chronic stroke, the use of FES also decreased the degree of subluxation ${ }^{(8-10,12)}$. Some researchers studied the effect of FES on the prevention of post-stroke shoulder subluxation, with the following parameters: electrodes on deltoid and supraspinal posterior fibers, frequency of $30 \mathrm{~Hz}$; wavelength of $300 \mu \mathrm{s} ; \mathrm{T}$-on of 06 seconds; T-off of 15 seconds, four times a day for four weeks, with a gradual duration between 30 and 60 minutes. They observed a significant decrease in shoulder pain and subluxation $(p<0.05)$ after the end of treatment, but at the 8-week follow-up there was no significant difference between the groups ${ }^{(28)}$. Other researchers made use of FES in the shoulder (supraspinal and deltoid) with a frequency of $30 \mathrm{~Hz}$; pulse duration time of $300 \mu \mathrm{s}$; T-on of 15 seconds; T-off of 10 seconds for 30 minutes, twice a week totaling ten sessions in three patients who had shoulder subluxation. The FES decreased pain, increased ROM from shoulder flexion and external rotation, increased motor function by the Fugl-Meyer scale and decreased shoulder subluxation, but this study did not use statistical methods to know if the results were significant ${ }^{(10)}$. A study performed with 120 patients who presented hemiplegia and sub-luxated shoulder provided treatment with conventional physiotherapy based on the concepts of Bobath and FES for 5 weeks ${ }^{(29)}$. Significant decrease in pain was observed both in post-treatment $(p<0.01)$ and in the follow-up of six months $(p<0.01)$ and in the follow-up of eighteen months $(p<0.01)$. There was also a decrease in shoulder subluxation posttreatment $(p<0.01)$, follow-up of six months $(p<0.05)$ and follow-up of eighteen months $(p<0.05)$.

\section{CONCLUSION}

Based on this study it can be concluded that FES associated with functional movements was effective in reducing the degree of subluxation of the shoulder joint and decreased pain in hemiparetic patients with glenohumeral subluxation after chronic stroke.

\section{AUTHOR'S CONTRIBUTION}

ROC and EWC elaborated the study design. WVS, GNM and EMF performed the data collection. WVS, ROC, EWC, JML and MPB performed the statistical analysis and the critical and intellectual revision of the manuscript. All authors read and approved the final manuscript.

\section{CONFLICTS OF INTEREST}

Nothing to declare

\section{AUTHOR DETAILS}

2 Departamento de Saúde Pública, Universidade Federal da Paraíba (UFPB), João Pessoa, Brasil.

${ }^{3}$ Departamento de Educação Física, Universidade Federal do Paraná (UFPR), Curitiba, Brasil.

\section{REFERENCES}

1. Sacco RL. Patogênese, classificação e epidemiologia das doenças vasculares cerebrais. In: Rowland LP, Pedley TA. Merritt, Tratado de neurologia. 11ำ edição. Rio de Janeiro: Guanabara Koogan. 2007;255-69.

2. O'Sullivan SB. Acidente vascular encefálico (AVE). In: O'Sullivan SB, Schmitz TJ. Fisioterapia, Avaliação e Tratamento. 5o edição. Barueri: Manole. 2010;763-834.

3. Lundy-Ekman L. Neurociência: Fundamentos para a Reabilitação. 3으 edição. São Paulo: Elsevier. 2008;477.

4. Brandão AD, Laskovski L, Garanhani MR. Estratégias de fisioterapia com enfoque na prevenção da dor no ombro de pacientes hemipléticos: revisão narrativa da literatura. Fisioter mov. 2008;21:71-8. 
5. Foongchomcheay A, Ada L, Canning C. Use of devices to prevent subluxation of the shoulder after stroke. Physiother Res Int. 2005;10:13445.

6. Ikeai T, Kenshaku T, Yoshiro K, Miyano S, Yonemoto K. Evaluation and treatment of shoulder subluxation in hemiplegia: relationship between subluxation and pain. Am J Phys Med Rehabil. 1998;77:421-426.

7. Doneux PS, Miyazaki ANM, Lemos PEG, Souza AS, Checchi. Tratamento da luxação recidivante anterior de ombro: uso de enxerto ósseo na deficiência da glenóide. Rev Bras Ortop. 1997;32:674-682.

8. Kobayashi H, Onishi H, Ihashi K, Yagi R, Handa Y. Reduction in subluxation and improved muscle function of the hemiplegic shoulder joint after therapeutic electrical stimulation. J Electromyogr Kinesiol. 1999;9:327336.

9. Paci M, Nannetti L, Rinaldi LA. Glenohumeral subluxation in hemiplegia: an overview. J Rehabil Res Dev. 2005;42:557-568.

10. Corrêa JP, Borges HC, Lucareli PR, Liebano RE. Estimulação elétrica funcional na subluxação crônica do ombro após acidente vascular encefálico: relato de casos. Fisioter Pesq. 2009;16:89-93.

11. Rezende FB, Borges HC, Monteiro VM, Masiero D, Chamlian TR. Efetividade da estimulação elétrica funcional no membro superior de hemiparéticos crônicos. Rev Neuroc. 2009;17:72-78.

12. Koyuncu E, Nakipoglu-Yuzer GF, Dogan A, Ozgirgin N. The effectiveness of functional electrical stimulation for the treatment of shoulder subluxation and shoulder pain in hemiplegic patients: A randomized controlled Trial. Disabil Rehabil. 2010;32:560-566.

13. Sonde L, Gip C, Fernaeus SE, Nilsson CG, Viitanen M. Stimulation with low frequency transcuteous electric nerve stimulation increases motor function of the post-stroke paretic arm. Stand J Rehabil Med. 1998;30:9599.

14. Metzker CAB. Tratamento conservador na síndrome do impacto no ombro. Fisioter mov. 2010;23:141-151.

15. Whiting WC, Zernicke RF. Biomecânica funcional e das lesões musculoesqueléticas. 2ㅇ edição. Rio de Janeiro: Guanabara Koogan. 2009;187.

16. Almeida OP. Mini Exame do Estado Mental e o diagnóstico de demência no Brasil. Arq Neuropsiquiatria. 2000;56:605-623.
17. Pimenta CAM, Teixeira MJ. Questionário de dor Mcgill: Proposta de adaptação para a língua portuguesa. Rev Bras Anestesiol. 1997;47:177186.

18. Marques AP. Manual de Goniometria. 2o edição. São Paulo: Manole. 2003;96.

19. Maki T, Emab Q, Cacho EWA, Paz LPS, Nascimento NH, Inoue MMEA, Viana MA. Estudo de confiabilidade da aplicação da escala de Fugl-meyer no Brasil. Rev bras fisioter. 2006;10:177-183.

20. Cacho EWA, Melo FRLV, Oliveira R. Avaliação da recuperação motora de pacientes hemiplégicos através do protocolo de desempenho físico FuglMeyer. Rev Neuroc. 2004;12:94-100.

21. Yu DT, Chae J, Walker ME, Fang ZP. Percutaneous intramuscular neuromuscular electric stimulation for the treatment of shoulder subluxation and pain in patients with chronic hemiplegia: a pilot study. Arch Phys Med Rehabil. 2000;82:20-25.

22. Duffau H. Brain plasticity: from pathophysiological mechanisms to therapeutic applications. J Clin Neurosci. 2006;13:885-897.

23. Nudo RJ. Mechanisms for recovery of motor function following cortical damage. Curr Opin Neurobiol. 2006;16:638-644.

24. Albert SJ, Kesserlring J. Neurorehabilitation of stroke. J Neurol. 2012;259:817-32.

25. Francisco GE, McGuire JR. Poststroke spasticity management. Stroke. 2012;43:3132-3136.

26. Dobkin BH. Strategies for stroke rehabilitation. Lancet Neurol. 2004,3:528536.

27. Wang RY, Chan RC, Tsai MW. Functional electrical stimulation on chronic and acute hemiplegic shoulder subluxation. Am J Phys Med Rehabil. 2000;79:358-390.

28. Linn SL, Granat MH, Lees KR. Prevention of shoulder subluxation after stroke with electrical stimulation. Stroke. 1999;30:963-968.

29. Chantraine A, Baribeault A, Uebelhart D, Gremion G. Shoulder pain and dysfunction in hemiplegia: effects of functional electrical stimulation. Arch Phys Med Rehabil. 1999;80:328-331. 